\title{
L368F/V408F double mutant of IBD of LEDGF/p75 retains interaction with M178I mutant of HIV-1 integrase
}

\author{
Anu George and Nidhanapati K Raghavendra* \\ Department of Biotechnology, Indian Institute of Technology Hyderabad, Kandi. Sangareddy- \\ 502285. Telangana. India. \\ * Correspondence: E-mail: raghunk@iith.ac.in; Tel: +91-40-2301-6054; Fax: +91-40-2301- \\ 6032 .
}

Abbreviations: IN: integrase; IBD: integrase binding domain; CCD: catalytic core domain

\begin{abstract}
Lens-epithelium-derived-growth factor (LEDGF/p75) is an essential host protein for integration of HIV-1 DNA into host genome. Earlier alanine scanning mutational analysis has revealed that residues I365, D366 and F406 in the integrase binding domain (IBD) of p75 are critical for interaction with HIV-1 integrase (IN), while K364, V408 have intermediate effect and residues N367, L368, R405, K407 show wild type binding with IN. To gain insight into contribution of side chains of L368 and V408 that are adjacent to critical residues I365 and F406, respectively, site directed mutation of these residues to Ile/Leu, Met and Phe has been performed and characterized in this study. In contrast to alanine substitution, L368F mutation showed a $\sim 25 \%$ decrease, while V408L and V408F showed wild type binding, to IN. Docking analysis of I365, D366 and F406 mutants of IBD with IN predicts that interaction between residue M178(IN) and I365(IBD) might lead to an encounter complex formation. Accordingly, M178I mutant of IN failed to interact with IBD. Interestingly, a L368F/V408F double mutant of IBD restored binding to M178I mutant of IN, indicating that altered hydrophobicity in the inter helical loops of IBD might make I365 more accessible for interaction with IN.

Keywords: HIV, integrase, LEDGF/p75, inter helical loops, M178, L368.

Introduction
\end{abstract}


HIV-1 integrase (IN) catalyses a two-step reaction to integrate viral DNA into human genome [14]. The first step is a removal of dinucleotide from 3`ends of viral DNA termed 3 processing and the second step termed as strand transfer involves the covalent bonding of the 3 ends into human genome. Several host proteins have been identified to be associated with IN and influence its activity [5-13]. Lens epithelium-derived growth factor (LEDGF/p75) is an essential host protein for integration of HIV-1 viral DNA into host genome. Extensive structural and biochemical studies of p75-IN interaction and its effect on activity of IN are known [14-25]. Two inter helical loops in Integrase Binding Domain (IBD) of p75 having residues, K364, I365, D366, N367, L368, R405, F406, K407 and V408 are the site of interaction with catalytic core domain (CCD) of IN $[16,17,21,25]$. Of these, I365, L368, F406 and V408 form a solvent accessible hydrophobic surface. Alanine scanning mutagenesis revealed that I365, D366 and F406 to absolutely critical for the interaction [16]. Substitution of K364 and V408 by alanine resulted in an intermediate binding.

Residue I365 of IBD is involved in hydrophobic interactions with the pocket created by side chains of L102, A128, W132 and M178 of CCD. In the complex, the delta carbon (CD1) of I365 rotates into the hydrophobic pocket of CCD [16,17]. In unbound state, CD1 of I365 is at $4.8^{\circ} \mathrm{A}$ from CD2 of L368, which increases to $9.1^{\circ} \mathrm{A}$ upon binding to IN and the distance between CD1 of I365 and CG1 of V408 increases from $6.2^{\circ} \mathrm{A}$ to $10.5^{\circ} \mathrm{A}$. The rotation of CG1-CD1 arm of I365 from hydrophobic pocket of IBD to that in IN dimer is critical for IBD-CCD interaction. Additionally, hydrophobic interactions are present between F406, V408 of IBD and W131 of CCD. A bidentate $\mathrm{H}$-bond involving oxygen of delta carbon of D366 (IBD) and main chain amine of E170 and H171 of CCD is essential for interaction. A salt bridge involving K364(IBD) \& E170(CCD) and hydrophobic interaction between V408(IBD) \& W131(CCD) provides stability to IBD-CCD complex.

Based on the IBD-CCD co-crystal structure, the steps leading to the rotation of side chain of I365, bidentate Hydrogen bond formation by D366 and hydrophobic interaction involving F406 of IBD cannot be derived. However, it is reasonable to assume that side chains of residues adjacent to the critical residues might provide clues to understanding an encounter complex formation [26]. The initial interactions during encounter complex formation between IN and IBD might trigger the side chain of I365 to lose contact with the hydrophobic surface comprising of side chains from L368, F406 and V408 [17] and gain interaction with the hydrophobic pocket of IN dimer comprising of L102, A128, A129, W132 (of chain B) and T174, M178 (of chain A). Mutagenesis of residues L368, V408 of IBD and M178 of IN performed in this study, combined with docking analysis, indicate that M178(IN)-I365(IBD) interaction could lead to IN-IBD encounter complex formation.

\section{Materials and Methods Mutagenesis}

Mutations were generated using overlap PCR protocol [27]. The plasmids pCP-GST-75-81 [28] and pINKB6HThr [14,29] were used as templates for mutagenesis of IBD and IN, respectively. All plasmids were sequence confirmed.

\section{Purification of recombinant proteins}

All proteins were induced in bacterial strain BL21(DE3)pLysS using $1 \mathrm{mM}$ IPTG at $28^{\circ} \mathrm{C}$ for $4 \mathrm{~h}$. His-tagged IN was purified as described earlier [29,30]. GST tagged IBD was purified as described earlier [28] except for use of buffer containing 500mM NaCl, 50mM NaPhosphate buffer (pH 6.5), $1 \mathrm{mM}$ EDTA, 5mM DTT and 0.2mM PMSF. Purified proteins were stored at $-20^{\circ} \mathrm{C}$ with $50 \%$ glycerol. Multiple batches of each protein were tested in the pull down assays. 


\section{In vitro pull-down assay}

The IN-IBD pull-down analysis [14,16,28,30-32] was performed in a phosphate pull-down buffer (PPD buffer) containing 50mM NaPhosphate buffer ( $\mathrm{pH}$ 6.5), $150 \mathrm{mM} \mathrm{NaCl}, 2 \mathrm{mM} \mathrm{MgCl} 2,25 \mathrm{mM}$ Immidazole, $0.1 \%$ NP-40. To $150 \mu 1$ of PPD buffer, $6 \mu \mathrm{g}$ of IN and $4.5 \mu \mathrm{g}$ of IBD were added and incubated for $10 \mathrm{~min}$ at $37^{\circ} \mathrm{C}$. Complexes were bound to Ni-sepharose-6-Fastflow beads (GE Healthcare) for $30 \mathrm{~min}$, at room temperature. Beads were washed with 5 volumes of PPD buffer prior to elution using $400 \mathrm{mM}$ Immidazole and resolved on a 10\% SDS-PAGE. Intensity of coomassie blue stained protein bands was quantified using Image Lab Software 4.0 (Bio-Rad).

\section{Computational analysis}

Building of mutants and docking was performed using Discovery Studio version 4.0 software (Accelrys, USA). Chains corresponding to IBD in IBD-CCD co-crystal structure 2B4J [17] were used for building the mutants and refined for side chains. IBD was docked onto CCD of 2B4J structure using ZDock program and poses generated were selected for further analysis based on proximity of any of IBD residues I365, D366, F406 and V408 to those of IN at the interface: L102, A128, A129, W131, W132, E170, H171, T174 and M178. Non-bond interactions at the interface of selected poses were compared to those reported in $2 \mathrm{~B} 4 \mathrm{~J}$.

\section{Statistical analysis}

Intensity of bands corresponding to IBD and IN were used to estimate the extent of complex formed. The IBD:IN ratio in sample having wt IBD was set to $100 \%$ and those having mutant IBD were calculated with respect to this. Data from more than three independent experiments has been used to obtain standard error.

\section{Results and Discussion}

\section{Phenylalanine at 368 position affects binding}

L368 of IBD has a hydrophobic interaction with A128 of IN [17]. L368A mutant of IBD was shown earlier [16] to not affect binding to IN (Fig 1, lane 5 and table 1). Since alanine scanning mutagenesis may not always reveal the significance of hydrophobic side chains [33], to probe if sulphur containing Methionine or bulky aromatic side chain of phenylalanine would affect binding to IN, L368M and L368F mutants of IBD were analysed. As shown in Fig 1, a decrease of $\sim 25 \%$ binding of L368F mutant (lane 3) to wt IN was observed compared to wt IBD (lane 1). L368M (lane 2) or L368I (lane 4) did not have any effect. The results (table 1) indicate that side chain of phenylalanine (F368) might distort the loop having critical residues I365 and D366 or alter the orientation of F406 having T-shaped pi-pi interaction with W131 of IN [17].

\section{Phenylalanine at 408 position retains wild type binding}

V408A mutant of IBD was shown to have an intermittent binding [16], here the binding of this mutant has been determined to be $76 \pm 1.55 \%$ of wt IBD (Fig 1, lane 10). V408M mutant showed $80 \pm 2.9 \%$ binding (lane 7), indicating that a sulphur-pi interaction [34,35] between M408 and F406 could interfere with F406-W131(IN) interaction resulting in a decrease in binding. In contrast, V408L (lane 6) or V408F (lane 9) mutant bound similar to wt IBD (table 1). Unlike, F368 that could alter orientation of F406 (lane 3), F408 (lane 9) might make stacking interaction with F406 [36] without disturbing F406-W131(IN) bond.

\section{F368 affects binding even in presence of F408}

To assess if presence of F408 could counter decreased binding of F368 mutant, L368F/V408F double mutant was analysed. However, as can be seen in Fig 1, L368F/V408F mutant showed $76 \pm 2.57 \%$ binding (lane 11) similar to that observed with L386F mutant, indicating that F368F406 interaction interferes with F406-W131(IN) bond even in presence of F408-F406 interaction. The presence of bulky side chains of phenylalanine at three of 4 residues in the hydrophobic 
surface do not seem to result in a severe structural distortion that would abolish IBD-IN complex formation. To analyse for an added effect [37], L368F/V408M mutant was analysed and found to have $73 \pm 2.9 \%$ binding (lane 12), similar to that of L368F mutant. Effect of M408 on orientation of F406 (lane 7) appears to be secondary to that at position 368. The binding of single and double mutants of IBD to wt IN summarized in table 1 indicates that interfering with orientation of F406 by side chain at 368 position can have an impeding effect on complex formation.

\section{Docking analysis predicts M178-I365 bond in encounter complex}

To gain insight into the role of the four residues I365, L368, F406 and V408 of hydrophobic surface in formation of complex with CCD, docking analysis of mutants of critical residues, namely, I365A, F406A and D366N [16,17] was performed. Table 2 summarizes the interactions observed in the docked complex of mutants of IBD with wt CCD, compared to those present in the 3D structure, 2B4J [17]. Two interactions are predicted in I365A(IBD)-CCD complex, with 7 interactions in F406A(IBD)-CCD complex and 13 interactions in D366N(IBD)-CCD complex. The interactions present in $2 \mathrm{~B} 4 \mathrm{~J}$ structure have been arranged in an order that reflects initial interactions (an encounter complex) between IBD and CCD at the top and subsequent interactions in the following rows of table 2 . The basis for this arrangement is the rationale that mutation of a critical residue (for eg., F406) would not significantly interfere with interactions occurring prior to those involving itself, but would abolish any interactions involving the residue (F406 in this example) as well as those subsequent to it. The interactions have been divided into 6 steps with each step having multiple interactions that cannot be predicted to be occurring in a particular order, but would occur prior to those in the next step. Accordingly, step 1 for IBD-IN interaction would involve A128(CCD)-L368(IBD) and M178(CCD)-I365(IBD) interaction, which might trigger step 2 involving W132(CCD)-I365(IBD) and W131(CCD)-F406(IBD) interactions. A similar pattern of increased interactions can be derived for step 3 to step 6, till a stable IBD-CCD complex is formed. It can be seen that hydrophobic interaction between alkyl side chains of I365(IBD) and M178(IN) is an interaction in step 1 (table 2) that is present in all the three mutant IBD-CCD docked complexes. Although these three mutants of IBD do not bind to CCD in pull-down assays [16], docking analysis has allowed identification of this interaction, as it can predict formation of complexes that are not biologically stable or are highly transient [38-40].

M178I mutant of IN does not bind to IBD

Based on the prediction that M178(IN)-I365(IBD) is an interaction for IN-IBD encounter complex formation (table 2), mutation of M178 of IN to isoleucine, so as to interfere with the extent of alkyl interaction with I365 of IBD, was designed. Isoleucine side chain is comparable to Methionine in bulkiness and alkyl nature [41], while being shorter by one Carbon-Carbon bond (C-C) length and lacking the sulphur atom. Towards forming an encounter complex, if M178(IN) has to initiate the rotation of $\mathrm{CD} 1$ of $\mathrm{I365}$, one $\mathrm{C}-\mathrm{C}$ shorter side chain of isoleucine could prove to be inadequate. To test this, M178I mutant of IN was generated and analysed for interaction with IBD. Since side chain of T174 is adjacent to M178 and is involved in carbon H-bonding [42,43] with D366 of IBD [17], T174V mutant of IN was also assayed. Valine lacks the hydroxyl group that imparts Hbonding ability to beta Carbon of T174 with delta O of D366(IBD). As shown in Fig 2, wt IBD showed a $65 \pm 3.2 \%$ complex formation with $\mathrm{T} 174 \mathrm{~V}$ mutant (lane 2, top panel) compared to that with wt IN (lane 1, top panel), probably reflecting a loss of Carbon H-bond between V174(IN) and D366(IBD).

In line with the prediction that M178(IN)-I365(IBD) interaction could be one of the first steps, wt IBD failed to yield complex with M178I mutant of IN (lane 2, bottom panel). Earlier, a M178C mutant of IN was shown to have a weak 3 'processing and no strand transfer activity [44], however, 
its interaction with IBD was not analysed. It was concluded that sulphur in the side chain of M178 is involved in a four-tiered pi-electron orbital interaction along with W132, F181 and F185 and that this interaction is critical only for tetramerization of IN required to catalyse strand transfer reaction. It is possible that M178I mutation analysed here fails to interact with IBD as the extent of hydrophobicity of the cleft formed between the monomers of IN is not sufficient for initiating the rotation of side chain of I365(IBD) residue observed in 3D structure, 2B4J [16,17].

\section{L368F/V408F double mutant of IBD interacts with M178I mutant of IN}

To test if altered hydrophobicity at the surface of IBD comprising of I365, L368, F406 and V408 might restore interaction with M178I mutant of IN, the single and double mutants of L368 and V408 of IBD were analysed for formation of complex. When compared to binding between wt IBD and T174V mutant of IN, it was observed that all the mutants of IBD, except for L386F/V408F double mutant, showed a trend similar (lower or wt level) to that observed with wt IN (Fig 2, top panel and table 1). Exceptionally, presence of three phenylalanine residues at positions 368, 406 and 408 (L368F/V408F double mutant) of IBD enhanced the complex formation (by $\sim 20 \%$ ) with T174V mutant of IN (Fig 2, top panel, lane 10) and enabled complex formation with M178I mutant of IN (Fig 2, bottom panel, lane 10). Similar to wt IBD, all other IBD mutants failed to interact with M178I mutant of IN (bottom panel). Based on the requirement for side chain of I365 to rotate from the hydrophobic surface of IBD to hydrophobic cleft of IN, it can be speculated from the results shown in Fig 2 that increase in hydrophobicity of the solvent accessible surface of L368F/V408F double mutant might make side chain of I365 available for interaction with IBD binding cleft of IN, even in the absence of a Methionine at 178 position that would initiate a rotation of side chain of I365.

In conclusion, deviating from the results of alanine scanning mutations performed earlier [16, 17], the mutation of V408(IBD) to phenylalanine or Leucine retained wild type levels of binding to IN, while phenylalanine at 368 position decreases the binding. The presence of phenylalanine at 368 or methionine at 408 position might interfere with orientation of side chain of adjacent residue F406 and consequently its critical interaction with W131 of CCD. Of significance is the loss of interaction between IN and IBD upon mutation of M178 to Isoleucine in CCD. The binding to M178I mutant of IN could be restored only by presence of phenylalanine at positions 368, 408 in addition to that at 406 (in $\mathrm{L} 368 \mathrm{~F} / \mathrm{V} 408 \mathrm{~F}$ double mutant). The study provides basis for encounter complex of IBD-IN with M178(IN)-I365(IBD) interaction initiating subsequent interface interactions. In the order of events resulting in the formation of a stable IBD-IN complex, F406(IBD)-W131(IN) interaction might be predicted to be followed by D366(IBD)E170/H171(IN) interaction. The L368F/V408F mutant of IBD and M178I mutant of IN can be used in structural analysis to reveal the various stages in formation of a final complex of IBD-IN. Such insight will help in understanding formation of protein-protein complexes and designing of small molecules that can alter solvent accessible hydrophobic surface of proteins to disrupt proteinprotein interactions [45-47].

\section{Acknowledgements}

The authors thank Alan Engelman (Harvard Medical School) for sharing plasmids expressing IN and IBD and Sree Hari Seenivasan for helping with designing of IN mutants. This work was supported by Department of Biotechnology (India) and ICMR (India) [grant no. BT/PR4843/MED/29/372/2012] to NKR.

\section{References}

[1] Asante-Appiah E, Skalka AM. HIV-1 integrase: structural organization, conformational changes, and catalysis. Advances in virus research. 52 (1999) 351-69. 
[2] Van Maele B, Debyser Z. HIV-1 integration: an interplay between HIV-1 integrase, cellular and viral proteins. AIDS rev. 7 (2005) 26-43.

[3] Craigie R, Bushman FD. HIV DNA integration. Cold Spring Harbor perspectives in medicine. 2 (2012) a006890.

[4] Engelman A, Cherepanov P. Retroviral integrase structure and DNA recombination mechanism. Microbiology spectrum. 2 (2014) 1.

[5] Kalpana GV, Marmon S, Wang W, et al. Binding and stimulation of HIV-1 integrase by a human homolog of yeast transcription factor SNF5. Science. 266 (1994) 2002.

[6] Farnet CM, Bushman FD. HIV-1 cDNA integration: requirement of HMG I (Y) protein for function of preintegration complexes in vitro. Cell. 88 (1997) 483-92.

[7] Chen H, Engelman A. The barrier-to-autointegration protein is a host factor for HIV type 1 integration. Proceedings of the National Academy of Sciences. 95 (1998) 15270-4.

[8] Cherepanov P, Maertens G, Proost P, et al. HIV-1 integrase forms stable tetramers and associates with LEDGF/p75 protein in human cells. Journal of Biological Chemistry. 278 (2003) 372-81.

[9] Van Maele B, Debyser Z. HIV-1 integration: an interplay between HIV-1 integrase, cellular and viral proteins. AIDS rev. 7 (2005) 26-43.

[10] Van Maele B, Busschots K, Vandekerckhove L, et al. Cellular co-factors of HIV-1 integration. Trends in biochemical sciences. 31 (2006) 98-105.

[11] König R, Zhou Y, Elleder D, et al. Global analysis of host-pathogen interactions that regulate early-stage HIV-1 replication. Cell. 135 (2008) 49-60.

[12] Poeschla EM. Integrase, LEDGF/p75 and HIV replication. Cellular and Molecular Life Sciences. 65 (2008) 1403-24.

[13] Raghavendra NK, Shkriabai N, Graham RL, et al. Identification of host proteins associated with HIV-1 preintegration complexes isolated from infected CD4+ cells. Retrovirology. 7 (2010) 66.

[14] Maertens G, Cherepanov P, Pluymers W, et al. LEDGF/p75 is essential for nuclear and chromosomal targeting of HIV-1 integrase in human cells. Journal of Biological Chemistry. 278 (2003) 33528-39.

[15] Llano M, Vanegas M, Fregoso O, et al. LEDGF/p75 determines cellular trafficking of diverse lentiviral but not murine oncoretroviral integrase proteins and is a component of functional lentiviral preintegration complexes. Journal of virology. 78 (2004) 9524-37.

[16] Cherepanov P, Sun ZY, Rahman S, et al. Solution structure of the HIV-1 integrase-binding domain in LEDGF/p75. Nature structural \& molecular biology. 12 (2005) 526-32.

[17] Cherepanov P, Ambrosio AL, Rahman S, et al. Structural basis for the recognition between HIV-1 integrase and transcriptional coactivator p75. Proceedings of the National Academy of Sciences of the United States of America. 102 (2005) 17308-13.

[18] Ciuffi A, Llano M, Poeschla E, et al. A role for LEDGF/p75 in targeting HIV DNA integration. Nature medicine. 11 (2005) 1287-9.

[19] Vandekerckhove L, Christ F, Van Maele B, et al. Transient and stable knockdown of the integrase cofactor LEDGF/p75 reveals its role in the replication cycle of human immunodeficiency virus. Journal of virology. 80 (2006) 1886-96.

[20] Llano M, Saenz DT, Meehan A, et al. An essential role for LEDGF/p75 in HIV integration. Science. 314 (2006) 461-4.

[21] Busschots K, Voet A, De Maeyer M, et al. Identification of the LEDGF/p75 binding site in HIV-1 integrase. Journal of molecular biology. 365 (2007) 1480-92. 
[22] Raghavendra NK, Engelman A. LEDGF/p75 interferes with the formation of synaptic nucleoprotein complexes that catalyze full-site HIV-1 DNA integration in vitro: implications for the mechanism of viral cDNA integration. Virology. 360 (2007) 1-5.

[23] Shun MC, Raghavendra NK, Vandegraaff N, et al. LEDGF/p75 functions downstream from preintegration complex formation to effect gene-specific HIV-1 integration. Genes \& development. 21 (2007) 1767-78.

[24] Pandey KK, Sinha S, Grandgenett DP. Transcriptional coactivator LEDGF/p75 modulates human immunodeficiency virus type 1 integrase-mediated concerted integration. Journal of virology. 81 (2007) 3969-79.

[25] Hare S, Shun MC, Gupta SS, et al. A novel co-crystal structure affords the design of gain-offunction lentiviral integrase mutants in the presence of modified PSIP1/LEDGF/p75. PLoS Pathog. 5 (2009) e1000259.

[26] Acuner Ozbabacan SE, Engin HB, Gursoy A, Keskin O. Transient protein-protein interactions. Protein Engineering, Design and Selection. 24 (2011) 635-48.

[27] Ho SN, Hunt HD, Horton RM, et al. Site-directed mutagenesis by overlap extension using the polymerase chain reaction. Gene. 77 (1989) 51-9.

[28] Cherepanov P, Devroe E, Silver PA, Engelman A. Identification of an evolutionarily conserved domain in human lens epithelium-derived growth factor/transcriptional co-activator p75 (LEDGF/p75) that binds HIV-1 integrase. Journal of Biological Chemistry. 279 (2004) 48883-92. [29] McKee CJ, Kessl JJ, Shkriabai N, et al. Dynamic modulation of HIV-1 integrase structure and function by cellular lens epithelium-derived growth factor (LEDGF) protein. Journal of Biological Chemistry. 283 (2008) 31802-12.

[30] Cherepanov P. LEDGF/p75 interacts with divergent lentiviral integrases and modulates their enzymatic activity in vitro. Nucleic acids research. 35 (2007) 113-24.

[31] Busschots K, Vercammen J, Emiliani S, et al. The interaction of LEDGF/p75 with integrase is lentivirus-specific and promotes DNA binding. Journal of Biological Chemistry. 280 (2005) $17841-7$.

[32] Rahman S, Lu R, Vandegraaff N, et al. Structure-based mutagenesis of the integraseLEDGF/p75 interface uncouples a strict correlation between in vitro protein binding and HIV-1 fitness. Virology. 357 (2007) 79-90.

[33] Zhao L, Tolbert WD, Ericksen B, et al. Single, double and quadruple alanine substitutions at oligomeric interfaces identify hydrophobicity as the key determinant of human neutrophil alpha defensin HNP1 function. PloS one. 8 (2013) e78937.

[34] Tatko CD, Waters ML. Investigation of the nature of the methionine- $\pi$ interaction in $\beta$-hairpin peptide model systems. Protein science. 13 (2004) 2515-22.

[35] Valley CC, Cembran A, Perlmutter JD, et al. The methionine-aromatic motif plays a unique role in stabilizing protein structure. Journal of Biological Chemistry. 287 (2012) 34979-91.

[36] Hunter CA, Singh J, Thornton JM. $\pi-\pi$ interactions: the geometry and energetics of phenylalanine-phenylalanine interactions in proteins. Journal of molecular biology. 218 (1991) 837-46.

[37] Wells JA. Additivity of mutational effects in proteins. Biochemistry. 29 (1990) 8509-17.

[38] Fernandez-Recio J, Totrov M, Skorodumov C, Abagyan R. Optimal docking area: a new method for predicting protein-protein interaction sites. PROTEINS: Structure, Function, and bioinformatics. 58 (2005) 134-43.

[39] Eyrisch S, Helms V. Transient pockets on protein surfaces involved in protein- protein interaction. Journal of medicinal chemistry. 50 (2007) 3457-64. 
[40] Ezkurdia I, Bartoli L, Fariselli P, et al. Progress and challenges in predicting protein-protein interaction sites. Briefings in bioinformatics. (2009) bbp021.

[41] Ohmura T, Ueda T, Hashimoto Y, Imoto T. Tolerance of point substitution of methionine for isoleucine in hen egg white lysozyme. Protein engineering. 14 (2001) 421-5.

[42] Scheiner S, Kar T, Gu Y. Strength of the $\mathrm{C} \alpha \mathrm{H} \cdot$ O hydrogen bond of amino acid residues. Journal of Biological Chemistry. 276 (2001) 9832-7.

[43] Horowitz S, Trievel RC. Carbon-oxygen hydrogen bonding in biological structure and function. Journal of Biological Chemistry. 287 (2012) 41576-82.

[44] Al-Mawsawi LQ, Hombrouck A, Dayam R, et al. Four-tiered $\pi$ interaction at the dimeric interface of HIV-1 integrase critical for DNA integration and viral infectivity. Virology. 377 (2008) 355-63.

[45] Christ F, Voet A, Marchand A, et al. Rational design of small-molecule inhibitors of the LEDGF/p75-integrase interaction and HIV replication. Nature chemical biology. 6 (2010) 442-8. [46] Kessl JJ, Jena N, Koh Y, et al. Multimode, cooperative mechanism of action of allosteric HIV-1 integrase inhibitors. Journal of Biological Chemistry. 287 (2012) 16801-11.

[47] Tsiang M, Jones GS, Niedziela-Majka A, et al. New class of HIV-1 integrase (IN) inhibitors with a dual mode of action. Journal of Biological Chemistry. 287 (2012) 21189-203.

Figure legends

Figure 1. Effect of mutations at L368 and V408 of IBD. Wild type (wt) or mutants of IBD were assayed for formation of complex with wt IN. The formed complexes were analyzed on a $10 \%$ SDS-PAGE gel. The mutant of IBD is indicated above the corresponding lane. Lanes from more than one gel have been assembled for purpose of representation.

Figure 2. Effect of T174V and M178I mutations of IN. Top and bottom panels (lanes 2-12) show interaction with T174V and M178I mutants of IN, respectively. Lane 1 shows interaction of wild type IN and IBD. Lane 2 has wt and lanes 3-12 show mutants of IBD as indicated above the lanes. Lanes from more than one gel have been assembled for purpose of representation.

\section{Table legends}

Table 1. Extent of IBD-IN complex formation. Mutants of IBD are indicated in the left most column, wild type IN in middle column and with T174V mutant of IN shown in right most column. Percent binding of IBD mutants with wt IN (middle column) and T174V mutant (right column) is indicated against each IBD mutant. The binding of wt IBD with wt IN or 174V mutant of IN is set to $100 \%$ for calculating binding of mutant IBD to respective IN.

Table 2. Interactions in docked complex of mutant IBD and wild type CCD. Interactions occurring between IBD and CCD are divided into six steps shown on the left. The details (column 2B4J) and type of interactions observed in 3D structure of IBD-CCD [17] are shown next. The interactions that were predicted in the docked complex of IBD mutants with wild type CCD are marked by a tick against those present in the 3D structure. Monomers of CCD are denoted by A, $\mathrm{B}$ and IBD by $\mathrm{C}$ chain in $3 \mathrm{D}$ structure(2B4J). * indicates a pi-alkyl interaction. 
Figure 1.

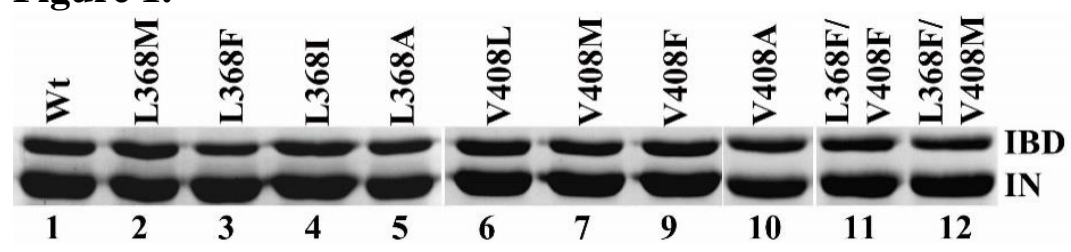

Figure 2.

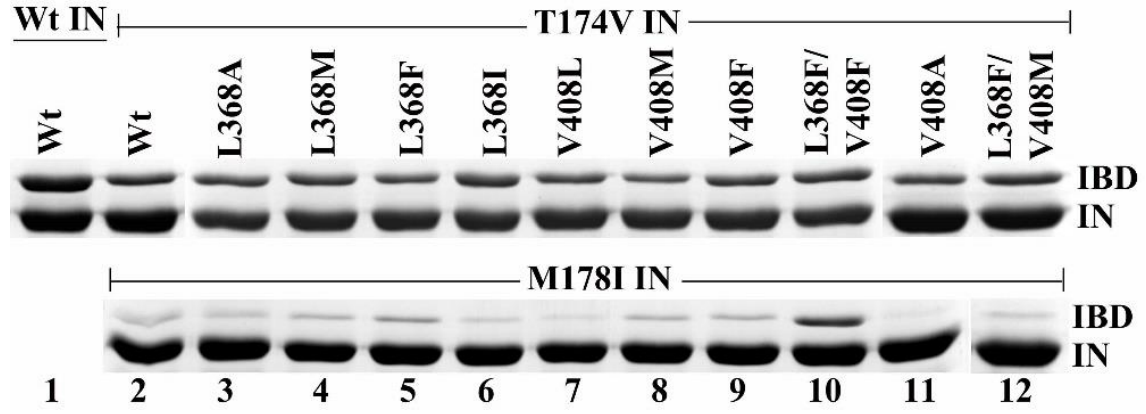

Table 1. Extent of IBD-IN complex formation.

\begin{tabular}{|c|c|c|}
\hline IBD mutant & Wt IN & T174V IN \\
\hline L368M & $97 \pm 3.10$ & $85 \pm 2.22$ \\
\hline L368F & $75 \pm 1.53$ & $66 \pm 3.12$ \\
\hline L368I & $98 \pm 5.55$ & $106 \pm 3.05$ \\
\hline L368A & $102 \pm 1.50$ & $105 \pm 4.86$ \\
\hline V408L & $90 \pm 2.43$ & $91 \pm 5.14$ \\
\hline V408M & $80 \pm 2.90$ & $73 \pm 3.50$ \\
\hline V408F & $92 \pm 2.79$ & $95 \pm 3.67$ \\
\hline V408A & $76 \pm 1.55$ & $84 \pm 4.00$ \\
\hline L368F/V408F & $76 \pm 2.57$ & $97 \pm 2.41$ \\
\hline L368F/V408M & $73 \pm 2.90$ & $83 \pm 2.90$ \\
\hline
\end{tabular}

Table 2. Interactions in docked complex of mutant IBD and wild type CCD.

\begin{tabular}{|c|l|c|c|c|c|}
\hline STEP & \multicolumn{1}{|c|}{ 2B4J } & Interaction & I365A & F406A & D366N \\
\hline \multirow{2}{*}{1} & B:ALA128 - C:LEU368 & Alkyl & $\sqrt{ }$ & & $\sqrt{ }$ \\
\cline { 2 - 6 } & A:MET178 - C:ILE365 & Alkyl & $\sqrt{ }$ & $\sqrt{ }$ & $\sqrt{ }$ \\
\hline \multirow{3}{*}{2} & B:TRP132 - C:ILE365 & Pi-Alkyl & & $\sqrt{ }$ & $\sqrt{ }$ \\
\cline { 2 - 5 } & B:TRP131 - C:PHE406 & Pi-Pi T-shaped & & $\sqrt{ } *$ & $\sqrt{ }$ \\
\cline { 2 - 5 } & C:LYS364:HA - A:GLN168:O & Carbon H-Bond & & $\sqrt{ }$ & $\sqrt{ }$ \\
\cline { 2 - 6 }
\end{tabular}




\begin{tabular}{|l|l|c|c|c|c|} 
& C:LYS360:HZ3 - A:ASP167:OD2 & Salt Bridge & & $\sqrt{ }$ & $\sqrt{ }$ \\
\hline \multirow{3}{*}{3} & A:THR174:HB - C:ASP366:OD2 & Carbon H-Bond & & $\sqrt{ }$ & \\
\hline & C:LYS407:HN - B:TRP131 & Pi-Donor H-Bond & & $\sqrt{ }$ & \\
\hline \multirow{5}{*}{4} & B:TRP131 - C:PHE406 & Pi-Pi T-shaped & & & $\sqrt{ }$ \\
\hline & B:ALA128 - C:ILE365 & Alkyl & & & $\sqrt{ }$ \\
\hline & B:LYS127 - C:VAL408 & Alkyl & & & $\sqrt{ }$ \\
\hline & B:ALA128 - C:VAL408 & Alkyl & & & $\sqrt{ }$ \\
\hline \multirow{3}{*}{5} & C:LYS364:HE2 - A:ASP167:O & Carbon H-Bond & & & $\sqrt{ }$ \\
\hline & B:TRP131:HE1 - C:ARG405:O & H-Bond & & & $\sqrt{ }$ \\
\hline & C:ILE365:HN - A:GLN168:O & H-Bond & & & $\sqrt{ }$ \\
\hline \multirow{5}{*}{6} & A:HIS171:NE2 - C:ASP366:OD2 & Attractive Charge & & & \\
\hline & A:GLU170:HN - C:ASP366:OD1 & H-Bond & & & \\
\hline & A:HIS171:HN - C:ASP366:OD2 & H-Bond & & & \\
\hline & A:HIS171:HD1 - C:ASP366:OD2 & H-Bond & & & \\
\hline
\end{tabular}

